\title{
Small Molecule Chemical Library
}

National Cancer Institute

\section{Source}

National Cancer Institute. Small Molecule Chemical Library. NCI Thesaurus. Code C16129.

Collection of small compounds (predominantly drug like organic substances synthesized using methods of combinatorial chemistry) used for screening for antineoplastic properties. 